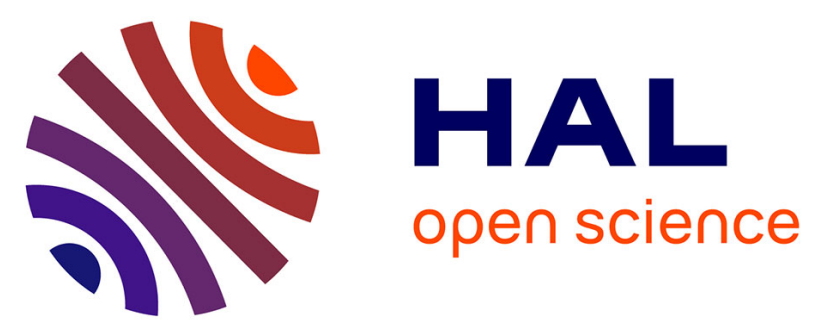

\title{
Croissance hivernale et printanière de prairies permanentes pâturées en montagne. II. - Variation de la croissance et de la composition chimique
}

\author{
Michel Duru, Roger Viard, Louis Sos
}

\section{- To cite this version:}

Michel Duru, Roger Viard, Louis Sos. Croissance hivernale et printanière de prairies permanentes pâturées en montagne. II. - Variation de la croissance et de la composition chimique. Agronomie, 1987, 7 (1), pp.51-59. hal-00884969

\section{HAL Id: hal-00884969 \\ https://hal.science/hal-00884969}

Submitted on 1 Jan 1987

HAL is a multi-disciplinary open access archive for the deposit and dissemination of scientific research documents, whether they are published or not. The documents may come from teaching and research institutions in France or abroad, or from public or private research centers.
L'archive ouverte pluridisciplinaire HAL, est destinée au dépôt et à la diffusion de documents scientifiques de niveau recherche, publiés ou non, émanant des établissements d'enseignement et de recherche français ou étrangers, des laboratoires publics ou privés. 


\title{
Croissance hivernale et printanière de prairies permanentes pâturées en montagne. II. - Variation de la croissance et de la compo- sition chimique
}

\author{
Michel DURU \\ avec la collaboration technique de Roger VIARD $\left(^{*}\right) \&$ Louis Sos $\left({ }^{*}\right)$
}

I.N.R.A., Station d'Agronomie (*) et Unité de Recherches sur les Systèmes Agraires et le Développement, Centre de Recherches de Toulouse, B.P. 27, F 31326 Castanet-Tolosan

Nous avons montré précédemment l'effet de la nutrition phosphopotassique et azotée sur la croissance du dactyle considéré comme plante témoin d'un couvert végétal pluri-spécifique. Ces résultats nous permettent d'interpréter les vitesses de croissance de 3 prairies permanentes différenciées du point de vue de leur flore et de leur fertilité phosphopotassique. Le pâturage est simulé mécaniquement par des prélèvements toutes les 3 semaines en moyenne en hiver et au printemps. Nous étudions ensuite dans quelle mesure on peut prévoir les variations de la composition chimique de l'herbe et donc la valeur alimentaire.

Avant début février, on note de faibles différences de vitesse de croissance selon la nutrition minérale (1 à $2,5 \mathrm{~kg}$ MS par ha et degré-jour). Les écarts deviennent par contre très élevés en fin d'hiver ( 3 à $13 \mathrm{~kg}$ MS par ha et degré-jour). Pour interpréter les variations saisonnières et interparcellaires des teneurs en azote ( 2 p. 100 à 4 p. 100) et en cellulose brute (12 p. 100 à 26 p. 100), nous émettons l'hypothèse du rôle de l'âge moyen des feuilles. Cet âge peut être évalué par la vitesse d'apparition des feuilles, elle-même dépendante de la nutrition minérale et du stade physiologique des graminées. La comparaison des résultats des situations pâturées réellement, à ceux obtenus par simulation montre que les écarts de composition chimique sont faibles et interprétables par les différences de fréquence de défoliation.

Mots clés additionnels : nutrition minérale, défoliation, température, azote minéral, azote soluble, azote nitrique, cellulose brute, valeur alimentaire.

Winter and spring growth of permanent pastures in mountains. II. Variation in growth rate and chemical composition.

Previously, we showed the effect of PK and N nutrition on the growth rate of cocksfoot chosen as an example of a multispecies canopy. This has allowed us to interpret the growth rate of 3 permanent pastures with different botanical composition and soil fertility level. Grazing was mechanically simulated by harvesting every three weeks in winter and spring. An attempt was made to forecast chemical composition and consequently feed value. Before mid-February, we observed only small differences in growth rate according to plant nutrition level (from 1 to $2.5 \mathrm{~kg} \mathrm{DM} \mathrm{ha}^{-1} \mathrm{~d}^{-1} \mathrm{C}^{-1}$ ). However, differences increased greatly after February (from 3 to $13 \mathrm{~kg} \mathrm{DM}$ $\mathrm{ha}^{-1} \mathrm{~d}^{-1} \mathrm{C}^{-1}$ ). The variation in nitrogen and cellulose content (respectively from $2 \%$ to $4 \%$ and from $12 \%$ to $26 \%$ ) between seasons and plots could probably be explained by a leaf age effect. Leaf age could be estimated by leaf appearance rate, which is dependent on plant nutrition and physiological stage of the grass. Comparison of results between plots subjected to real or simulated grazing showed that differences in chemical composition were low and interpretable by differences in defoliation frequency.

Additional key words : mineral nutrition, defoliation, temperature, mineral nitrogen, soluble nitrogen, nitrate nitrogen, cellulose, feeding value. 


\section{INTRODUCTION}

L'effet du pâturage des animaux en période de faible croissance de la végétation est peu connu tant du point de vue du couvert végétal que des prélèvements réalisés et de leur valeur alimentaire. Dans les Pyrénées Centrales, en fin d'hiver, une estimation des quantités ingérées a pu être réalisée à partir de l'étude du comportement alimentaire des animaux (GIBON \& BALENT, 1984). Les observations d'un couvert végétal soumis à une simulation mécanique du pâturage toutes les trois semaines ont montré de fortes variations de la croissance selon la nutrition minérale et le climat. Les teneurs en azote et cellulose brute de ces prélèvements sont variables mais laissent supposer des valeurs alimentaires bien supérieures aux foins distribués en hiver (DURU, 1983).

L'objet du travail présenté est de préciser ces facteurs de variation et dans une certaine mesure, d'étudier les possibilités de prévision de leurs effets sur la croissance et la composition chimique.

En premier lieu, nous évaluerons, par simulation du pâturage, la croissance potentielle en hiver et au début de printemps ainsi que les niveaux de valorisation permis selon la nutrition minérale (azotée et phosphopotassique). Les variations de composition chimique des prélèvements seront interprétées en relation avec la morphogenèse des graminées. En second lieu, les résultats de croissance et de composition chimique seront confrontés aux observations réalisées sur les couverts réellement pâturés. Il s'agit d'analyser dans quelle mesure la défoliation que nous avons pratiquée d'une part sur- ou sous-estime les prélèvements réalisés par les animaux, et d'autre part modifie la composition chimique des disponibilités sur pied.

\section{MATÉRIEL ET MÉTHODES}

Les caractéristiques des prairies permanentes étudiées ont été présentées dans l'article précédent
(DURU, 1987a). Elles sont brièvement rappelées dans la légende du tableau 1. Dans la suite du texte, nous caractériserons ces 3 parcelles par les initiales suivantes : $\mathrm{B}$ bonne, $\mathrm{M}$ moyenne, $\mathrm{C}$ carencée. Les températures moyennes $(-10 \mathrm{~cm}$ dans le sol) ont été approximativement de 8 degrés en novembre, 4 en décembre, 2 en janvier, 3 en février, 5 en mars et 8 en avril. L'enneigement au sol a varié de 47 à 66 jours pour les 4 années d'étude.

Sur chacune des parcelles, les prélèvements ont eu lieu toutes les 3 semaines sur les mêmes placettes, (ou moins souvent en cas de couverture neigeuse) pendant la période de pâturage soit de début novembre à fin avril et durant 4 hivers consécutifs.

3 modalités différentes d'apport d'azote ont été testées sur les parcelles B et $\mathbf{M}$ (cf. tabl. 1).

\section{A. Mesures de biomasse}

Elles ont été réalisées sur des placettes de $0,5 \mathrm{~m}^{2}$ avec 4 répétitions par traitement, ces placettes étant mises en défens. La technique de prélèvement doit être adaptée aux petites quantités (jusqu'à $20 \mathrm{~kg}$ de MS $\mathrm{ha}^{-1}$ ). Elle a été décrite précédemment (DuRU, 1983).

\section{B. Composition chimique}

La composition chimique a été réalisée comme base de prévision de la valeur fourragère : analyses d'azote (méthode KJELDAHL) sur chaque échantillon, analyses de cellulose brute (méthode de WEENDE), analyses d'azote soluble et nitrique certaines années.

Des prélèvements, en dehors des mises en défens, donc sur des prairies pâturées, ont été réalisés dans un petit nombre de situations ( 13 cas en tout) afin de comparer d'une part notre niveau de défoliation à celui des troupeaux et d'autre part la composition chimique du couvert pâturé en continu à celle des repousses que nous avons prélevées.

TABLEAU

Fertilisation selon les parcelles et les années.

$B, M, C$ : parcelles. Pour l'horizon 0-15 cm du sol, nous avons respectivement $0,108 \% ; 0,045 \% ; 0,018 \%$ d'acide phosphorique; $0,051 \% ; 0,030 \% ; 0,009 \%$ de potasse échangeable.

No: pas de fertilisation azotée; $N 0+60: 60 \mathrm{~N}$ début mars; $N 60+60: 60 \mathrm{~N}$ à l'automne et début mars.

Fertilization according to plots and years.

$B, M, C$ : plots. For $0-15 \mathrm{~cm}$ soil horizon, we had respectively $0.0108 \% ; 0.0045 \% ; 0.0018 \%$ of phosphoric acid ; $0.0051 \% ; 0.0030 \% ; 0.0009 \%$ of potash. N0: no nitrogen fertilization

$\mathrm{NO}+60: 60 \mathrm{~kg}$ of nitrogen at the beginning of March; $\mathrm{N} 60+60: 60 \mathrm{~kg}$ of nitrogen in autumn and at the beginning of March.

\begin{tabular}{|c|c|c|c|c|c|c|c|}
\hline & & B & & & $\mathbf{M}$ & & C \\
\hline & No & $\mathrm{N} 0+60$ & $N 60+60$ & No & $\mathrm{NO}+60$ & $N 60+60$ & No \\
\hline $81 / 82$ & $x$ & & & $\mathbf{x}$ & & & $x$ \\
\hline $82 / 83$ & $\mathrm{x}$ & $\mathrm{x}$ & & $\mathrm{x}$ & $\mathrm{x}$ & & $\mathrm{x}$ \\
\hline $83 / 84$ & $x$ & $\mathrm{x}$ & $\mathrm{x}$ & $\mathrm{x}$ & $\mathrm{x}$ & $\mathbf{x}$ & $\mathrm{x}$ \\
\hline $84 / 85$ & $\mathrm{x}$ & $\mathrm{x}$ & $\mathrm{x}$ & $\mathrm{x}$ & $\mathrm{x}$ & $\mathrm{x}$ & $\mathrm{x}$ \\
\hline
\end{tabular}




\section{RÉSULTATS}

\section{A. Croissance}

Sur la figure 1, nous avons représenté les productions cumulées des prélèvements de matière sèche au cours des hivers de façon à comparer facilement les traitements 2 à 2 : B60 (abscisse positive)/B0 (ordonnée positive) : $\mathrm{M} 0 / \mathrm{C} 0, \mathrm{C} 0 / \mathrm{B} 60 \ldots$, le traitement $\mathrm{M} 60$ n'apparaît pas. Les variations de longueur des segments joignant chaque point permettent de visualiser les variations saisonnières de croissance. Les productions cumulées varient selon les années de 700 à $1100 \mathrm{~kg} \mathrm{MS} / \mathrm{ha}$ pour la parcelle $\mathrm{C}$, à 2900 à $3050 \mathrm{~kg}$ pour la parcelle $\mathrm{B}$ avec azote. En début d'hiver, les quantités prélevées sont souvent très voisines entre parcelles, et ce même pour les situations recevant de l'azote à l'automne. Les effets parcelles et azote sont toujours importants en fin d'hiver : c'est pour les 3 dernières observations que les points se détachent très nettement des bissectrices. Les accroissements de matière sèche ne sont importants que pour les 2 derniers contrôles, c'est-à-dire en mars et avril.

Sur la figure 2, nous avons représenté les vitesses de croissance journalière en fonction de la température du sol. Pour chaque traitement, de même que pour l'étude de l'écophysiologie du dactyle (DURU, 1987a), les données antérieures ou postérieures à mi-février ont été symbolisées différemment et considérées séparément pour les calculs.

Le coefficient a de la relation MS $\mathrm{j}^{-1}=\mathrm{a}(\theta+\mathrm{b})$ représente la croissance par degré et par jour (tabl. 2). On note qu'à l'automne, ces coefficients sont très voisins pour les parcelles et que l'effet de l'azote n'apparaît pas. Par contre, après mi-février, les écarts sont très importants selon les parcelles : $2,7 \mathrm{~kg}$ de MS par degré-jour en $C$ contre 7,0 en $B$. Il en est de même pour l'effet de la nutrition azotée qui permet presque de doubler la vitesse de croissance pour la parcelle B. Nous pouvons noter sur la figure 2 que les points de fin d'hiver 1985 avec azote correspondent à des vitesses de croissance plus faibles, à température égale, que ceux des autres années. Il se peut que, dans ce cas, la nutrition azotée ait été insuffisante du fait d'une plus faible minéralisation de l'azote organique du sol.

Pour la période postérieure à mi-février, la vitesse de croissance observée de $13 \mathrm{~kg}$ de MS par degré-jour (température du sol) est apparemment supérieure à celle obtenue pour le déroulement complet du cycle reproducteur : $10 \mathrm{~kg}$ de MS par degré-jour (température de l'air) (DURU, 1985b). Sachant qu'en fin

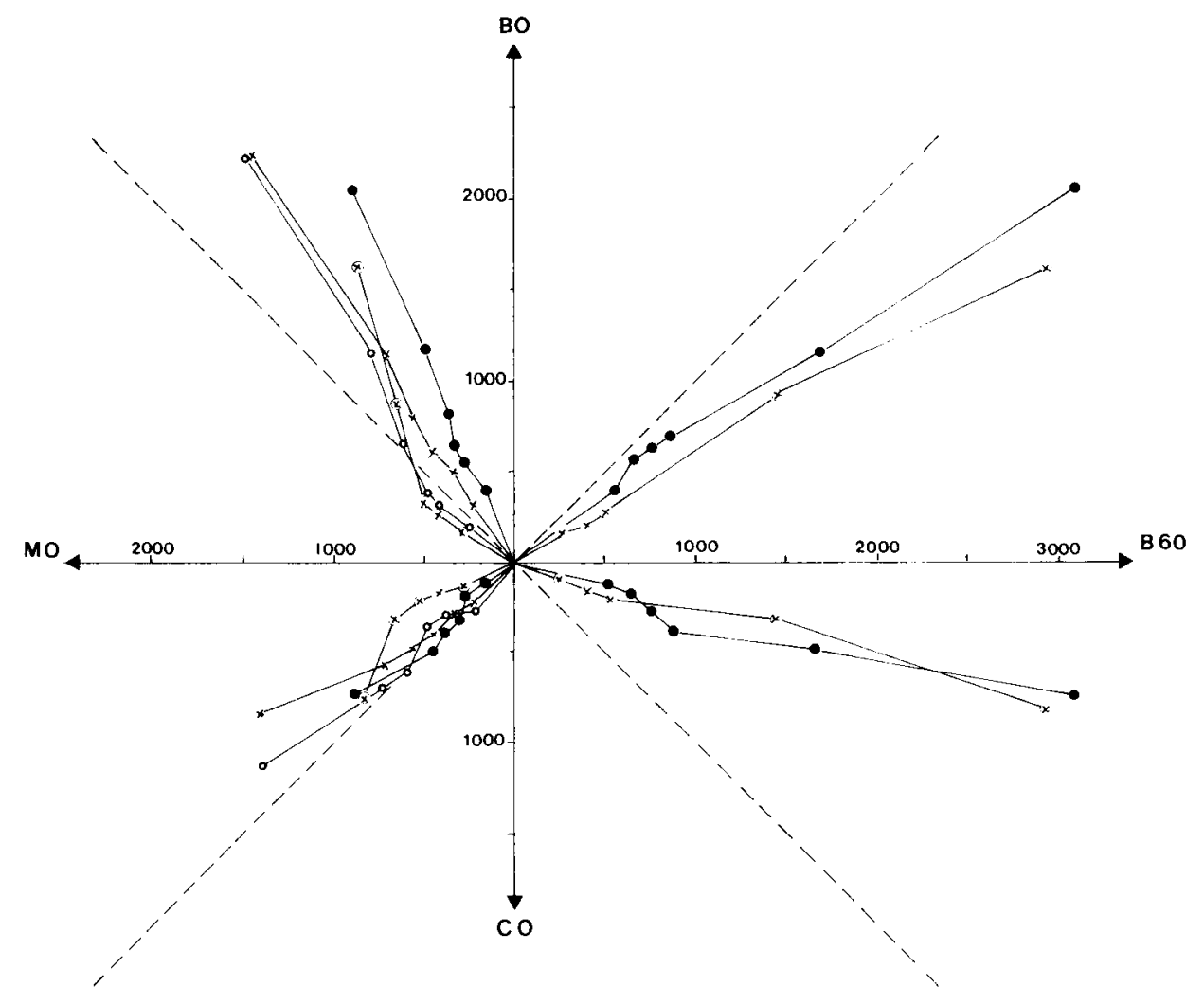

Figure 1

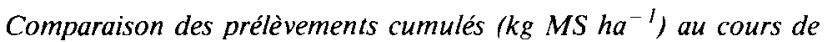
l'hiver selon les parcelles et les années. B60: parcelle $B$ avec $60 \mathrm{~kg}$ d'azote début novembre et début mars; $B O$ : parcelle $B$ sans azote ; MO : parcelle $M$ sans azote; $C 0$ : parcelle $C$ sans azote. $x$ hiver $81 / 82$; $\bigcirc$ hiver $82 / 83$; $\otimes$ hiver $83 / 84$; hiver $84 / 85$.

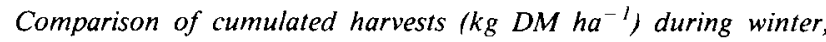
according to plots and years. B60, plot B with $60 \mathrm{~kg}$ of nitrogen at the beginning of November and March; BO, plot B without nitrogen; $M 0$ plot $M$ without nitrogen; $C 0$, plot $C$ without nitrogen. $x$ winter $81 / 82 ; \bigcirc$ winter $82 / 83$; $\otimes$ winter $83 / 84$; $\bullet$ winter $84 / 85$. 


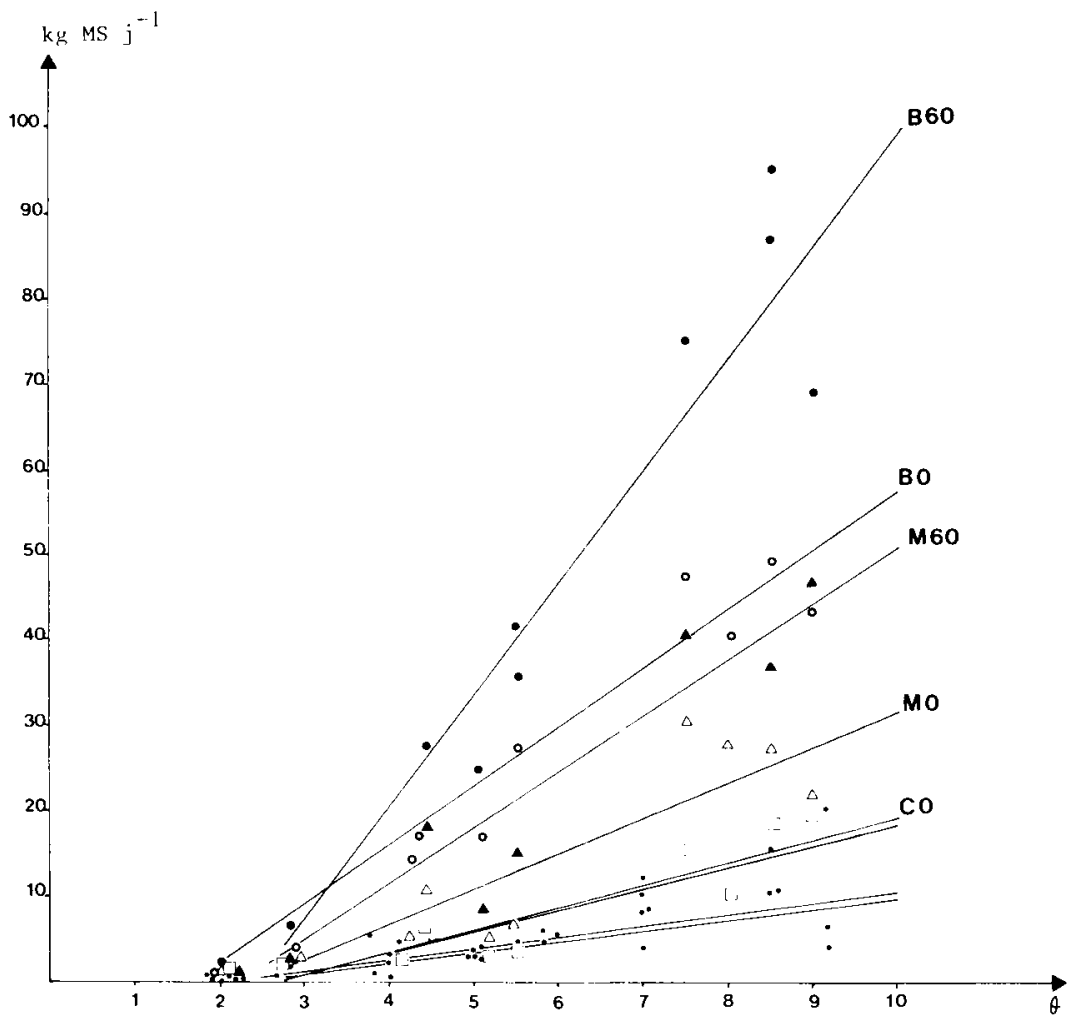

Figure 2

Relation entre la vitesse de croissance et la température du sol $(10 \mathrm{~cm})$; données de toutes les parcelles avant mi-février ( . );B60 (๑), BO (O), $M 60(\mathbf{\Delta}), M O(\wedge), C O(\square)$ après mi-février.

\section{TABLEAU 2}

Valeur des coefficients de corrélation et de régression entre la vitesse de croissance et la température au sol: $M S j^{-1}=a(\theta+b)$. Tous les coefficients de corrélation sont significatifs au seuil 0,001 sauf pour B60 avant mi-février.

Correlation coefficient values, and regressions between growth rate and soil temperature : $D M j^{-1}=a(\theta+b)$. All correlation coefficients were significant $(0,001)$ except $B 60$ before mid-February.

\begin{tabular}{crccc}
\hline Traitements & $\mathrm{a}$ & $\mathrm{b}$ & $\mathrm{r}$ & $\begin{array}{c}\text { Nombre de } \\
\text { donnees }\end{array}$ \\
\hline Avant mi-février & & & & \\
B0 & 2,46 & $-2,43$ & 0,896 & 13 \\
M0 & 1,29 & $-1,55$ & 0,796 & 13 \\
C0 & 1,19 & $-0,95$ & 0,714 & 13 \\
B60 & 2,48 & $-1,92$ & 0,936 & 6 \\
Après mi-février & & & & \\
B0 & 7,02 & $-1,92$ & 0,968 & 10 \\
M0 & 4,25 & $-2,42$ & 0,878 & 10 \\
C0 & 2,73 & $-2,54$ & 0,900 & 10 \\
B60 & 13,03 & $-2,41$ & 0,948 & 11 \\
M60 & 6,71 & $-1,93$ & 0,949 & 8 \\
\hline \hline
\end{tabular}

d'hiver, la température du sol est toujours inférieure à celle de l'air, nous avons vérifié que ces résultats sont en fait proches.

\section{B. Composition chimique}

\section{Azote total et cellulose brute}

Compte tenu du faible nombre de données en début et milieu d'hiver du fait de l'enneigement, nous avons
Relationship between growth rate and soil temperature $(10 \mathrm{~cm})$; all

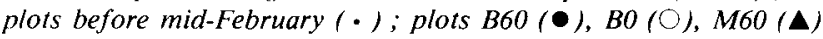

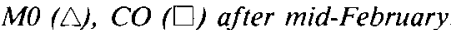

regroupé celles des mois de novembre et décembre, ainsi que février et mars, de façon à avoir au moins 3 données par traitement. Les valeurs moyennes et écarts types des teneurs en cellulose et en azote sont indiqués respectivement dans les tableaux 3 et 4 . Nous constatons que dans tous les cas, la teneur en azote diminue en début d'hiver puis remonte à partir de février-mars. Le maximum est atteint en début avril puis les teneurs stagnent ou baissent. A chaque période, nous observons que les teneurs vont en croissant avec la fertilité de la parcelle. Lorsqu'il y a apport d'azote, les teneurs sont toujours très supérieures. Pour la cellulose, nous observons des évolutions inverses.

En première approximation, la teneur en cellulose dépend de l'âge des feuilles surtout lorsqu'on se rapproche de leur sénescence puisque le contenu cellulaire disparaissant, il ne reste plus que les membranes (GIL LET, 1980). Nous émettons l'hypothèse que les variations observées dans les teneurs en cellulose peuvent s'interpréter en partie par des différences d'âge moyen des feuilles. Pour le dactyle présent dans le couvert végétal, nous avons évalué cet âge indirectement par le rapport suivant :

feuilles vertes déjà présentes à l'observation $t_{n}-1$ nombre total de feuilles vertes présentes à l'observation $t_{n}$

Plus ce rapport est élevé, plus l'âge moyen est important. Sur la figure 3, nous avons représenté l'évolution de ce rapport au cours de l'hiver, (rapport des longueurs cumulées pour l'hiver $82 / 83$ et rapport de poids de feuilles pour l'hiver $84 / 85$ ). Le rapprochement des données de la figure 3 et du tableau 3 est à 
TABLEAU 3

Moyennes (m), écarts types ( $t)$ des teneurs en cellulose brute (\% MS) selon la période de l'année et les parcelles (I seule donnée pour le mois de janvier).

Cellulose content (\% DM), means ( $m$ ) and standard deviations ( $t$ ) according to seasons and plots (only one result for January).

\begin{tabular}{cccccccccccc}
\hline \hline Mois & nov. & déc. & & jan. & & fév. & mars & & déb. avr. & fin avr. \\
\hline & $\mathrm{m}$ & $\mathrm{t}$ & $\mathrm{m}$ & 1 & $\mathrm{~m}$ & $\mathrm{t}$ & $\mathrm{m}$ & $\mathrm{t}$ & $\mathrm{m}$ \\
\hline B0 & 17,9 & 0,9 & 20,2 & - & 11,7 & 3,1 & 12,9 & 1,4 & 14,3 \\
M0 & 21,6 & 0,5 & 23,3 & - & 16,7 & 3,2 & 14,6 & 1,4 & 15,5 & 1,4 \\
C0 & 24,6 & 1,2 & 25,0 & - & 16,4 & 2,5 & 18,7 & 2,8 & 17,7 \\
\hline
\end{tabular}

TABLEAU 4

Moyennes (m), écarts types ( $t$ ) des teneurs en azote total (\% MS) selon la période de l'année et les traitements. Nitrogen content (\% DM), means $(m)$ and standard deviations ( $t$ ) according to seasons and plots.

\begin{tabular}{ccccccccccccc}
\hline Mois & nov. & déc & & jan. & & fév. & mars & & déb. avr. & fin avr. \\
\hline & $\mathrm{m}$ & $\mathrm{t}$ & $\mathrm{m}$ & $\mathrm{t}$ & $\mathrm{m}$ & $\mathrm{t}$ & $\mathrm{m}$ & $\mathrm{t}$ & $\mathrm{m}$ & $\mathrm{t}$ \\
\hline B0 & 2,8 & 0,3 & 2,3 & 0,2 & 3,0 & 0,2 & 3,9 & 0,7 & 3,7 & 0,1 \\
M0 & 2,4 & 0,3 & 2,3 & 0,05 & 3,1 & 0,2 & 3,6 & 0,5 & 3,4 & 0,05 \\
C0 & 2,3 & 0,3 & 2,1 & 0,3 & 2,6 & 0,2 & 3,1 & 0,5 & 3,4 & 0,3 \\
B60 & 3,7 & 0,6 & - & - & 3,2 & 0,05 & 4,8 & 0,1 & 3,9 & 0,2 \\
M60 & - & - & - & - & - & - & 4,7 & 0,4 & 4,0 & 0,05 \\
\hline
\end{tabular}

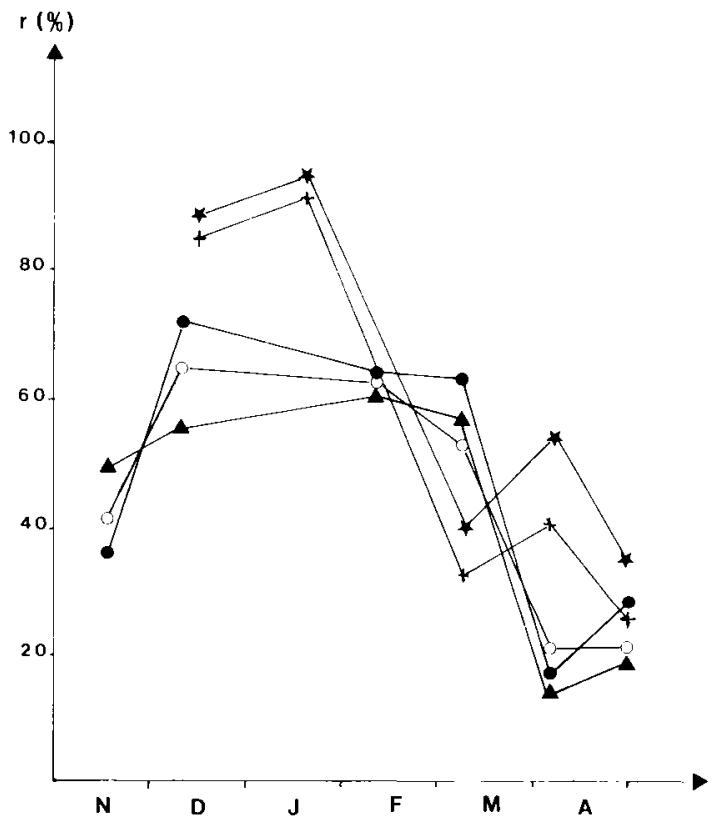

Figure 3

Pour le dactyle présent dans le couvert végétal, évolution du rapport en fonction des mois :

$r=\frac{\text { feuilles vertes déjà présentes à } t_{n-1}}{\text { nombre total de feuilles vertes présentes à } t_{n}}$;

rapport de longueur pour l'hiver 82/83, parcelles BON (+) et MON $(\star)$; rapport de poids pour l'hiver $84 / 85$, parcelles $B 0 N$ (O), $B 60 \mathrm{~N}(\bullet), M 60 \mathrm{~N}(\mathbf{\Delta})$.

For cocksfoot in the canopy, change in the ratio according to months :

$r=$ green leaves previously observed at $t_{n-1}$ total number of leaves observed at $t_{n}$

length ratio for plots $B O N(+), M O N(\star)$, winter $82 / 83$; weight ratio for plots $B 0 N(O), B 60 N(\bullet), M 60 N(\Delta)$, winter $84 / 85$. réaliser avec prudence puisqu'il s'agit dans un cas du dactyle et dans l'autre de la flore dans sa diversité, même si le dactyle y est prépondérant pour les parcelles $B$ et $M$. Cette réserve étant faite, on note une même tendance saisonnière rendant plausible l'hypothèse considérée. Les variations du rapport défini ci-dessus peuvent s'interpréter par des variations du rythme d'apparition des feuilles: au milieu de l'hiver, le rythme d'apparition étant très bas, la part des feuilles déjà présentes à l'observation $t_{n}-1$ est alors plus élevée qu'en début d'hiver et a fortiori qu'au printemps (DURU, 1987a). Cet effet est renforcé lorsque la nutrition phospho-potassique est limitante.

Les variations de teneurs en azote sont plus compliquées à interpréter puisqu'elles dépendent en partie du niveau de nutrition azoté que nous ne connaissons pas. Néanmoins, la teneur en azote des feuilles de graminées baisse avec leur âge, même avant leur sénescence ou complète élongation (ROBSON \& DEACON, 1978). D'ailleurs, pour l'hiver $84 / 85$, nous avons trouvé que la teneur en azote de feuilles de dactyle apparues entre $t_{n-1}$ et $t_{n}$ est en moyenne 15 p. 100 supérieure à celles déjà présentes à $t_{n-1}$ (moyenne de 12 observations). Compte tenu par ailleurs de la liaison observée entre teneurs en cellulose et en azote (fig. 4), nous pensons que l'âge moyen des feuilles est une des hypothèses vraisemblables pour expliquer les variations de teneurs en azote. Toutefois, lorsque les vitesses de croissance s'élèvent en fin d'hiver, les phénomènes de dilution mis en évidence par SALETTE \& LEMAIRE (1981) peuvent expliquer l'abaissement des teneurs observé presque systématiquement entre début et fin avril (tabl. 4). 


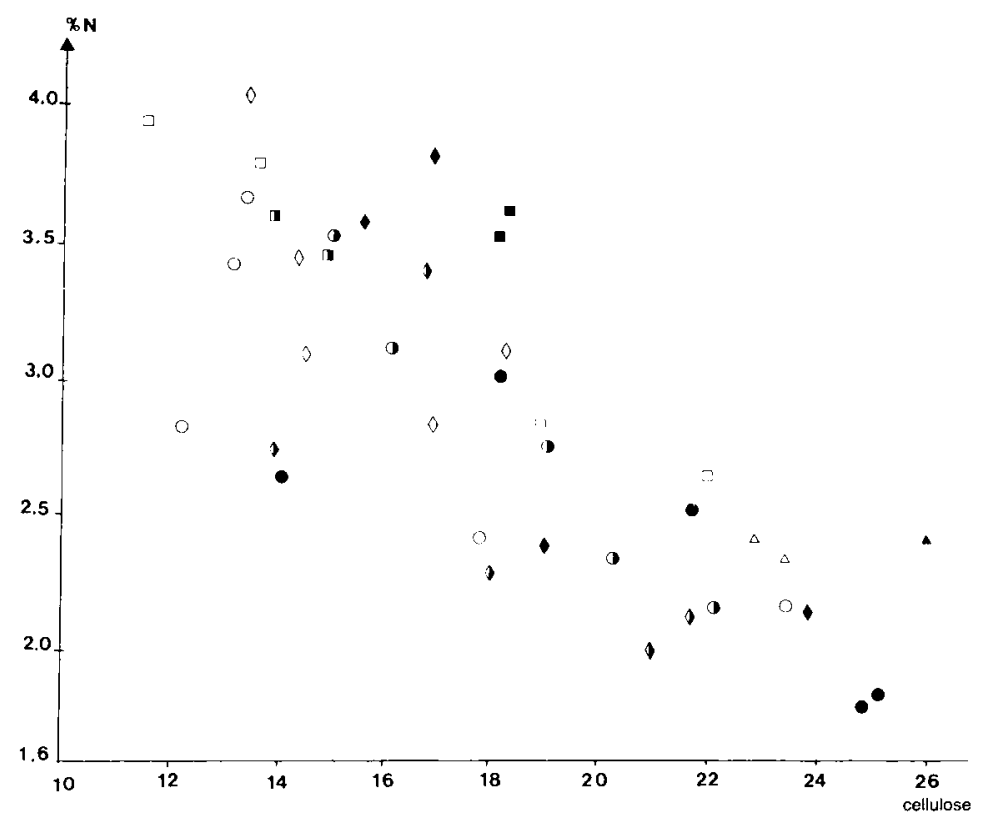

Figure 4

Variations comparées des teneurs en azote et cellulose (p. 100 pour les placettes ne recevant pas de fertilisation azotée; $0,0,-p o u r$ l'hiver $81 / 82$ (respectivement pour $B, M, C) ; \square, \square, \square$ hiver $82 / 83 ; \triangle, \Delta, \boldsymbol{\Delta}$ hiver $83 / 84 ; \diamond, \triangleleft, \bullet$ hiver $84 / 85$.

\section{Azote soluble et azote nitrique}

Sur la figure 5 sont indiquées les teneurs en azote total et soluble des prélèvements de début de prin-

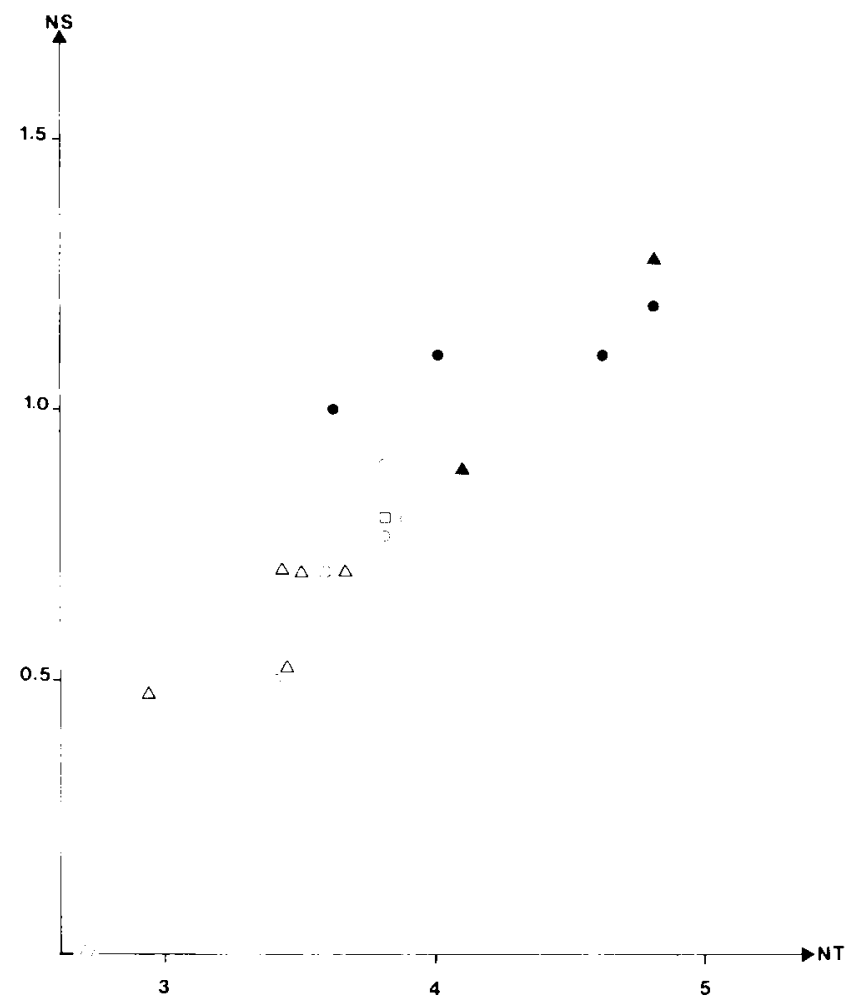

Figure 5

Relation entre azote soluble (Ns) et azote total (Nt). O BO, $-B 60$, $\triangle M O, \triangle M 60, \square C O$.

Relationship between soluble nitrogen (Ns) and total nitrogen $(N t)$. $\bigcirc B 0, \bullet B 60, \triangle M 0, \triangle M 60, \square C O$
Comparison of nitrogen and cellulose variation (\% DM) for plots without nitrogen fertilization ; $\bigcirc, 0,-$ winter $81 / 82$ (respectively for $B, M, C) \square, \square, \square$ winter $82 / 83 ; \Delta, \boldsymbol{\Lambda} ; \boldsymbol{\Delta}$ winter $83 / 84 ; \diamond$, $\checkmark$ winter $84 / 85$.

temps. La teneur en azote soluble croît avec la teneur en azote total mais le rapport azote soluble/azote total (Ns/Nt) croît avec l'enrichissement en azote total (17 à 28 p. 100). La fertilisation azotée en fin d'hiver augmente proportionnellement plus la teneur en azote soluble qu'en azote total. D'une manière générale, le rapport $\mathrm{Ns} / \mathrm{Nt}$ est inférieur à celui observé couramment pour des premiers cycles (SALETTE $e t$ al., 1984) et pour tous les cycles de ces mêmes parcelles (DuRU, non publié).

Les teneurs en azote nitrique sont minimales en milieu d'hiver (tabl. 5). Elles atteignent les valeurs les plus élevées pour la coupe suivant un apport d'azote $(B 60+60$ le 14/11; B60 + 60 et M0 + 60 le 9/4). Cependant, nous observons qu'elles sont très faibles et toujours inférieures au seuil considéré comme critique $(0,25 \mathrm{p} .100)$ et ce, même en présence de fertilisation azotée et pour des stades très précoces d'utilisation de l'herbe.

\section{TABLEAU 5}

Teneur en azote nitrique (\% MS) selon les parcelles et la fertilisa tion azotée (hiver 84/85).

Nitrate content (\% DM) according to plots and nitrogen fertilization (winter 84/85).

\begin{tabular}{lcccccc}
\hline & $14 / 11$ & $10 / 12$ & $13 / 2$ & $7 / 3$ & $9 / 4$ & $29 / 4$ \\
\hline B60 +60 & 0,058 & 0,019 & 0,017 & 0,015 & 0,038 & 0,026 \\
B0 & 0,019 & 0,015 & 0,0 & 0,016 & 0,016 & 0,019 \\
M0 60 & - & - & - & - & 0,070 & 0,020 \\
M0 & 0,012 & 0,011 & 0,0 & 0,037 & 0,024 & 0,020 \\
C0 & 0,012 & - & 0,010 & 0,0 & 0,014 & 0,013 \\
\hline
\end{tabular}




\section{DISCUSSION}

\section{A. Variabilité de la croissance d'hiver et de début de printemps}

D'une manière générale, les données sur la croissance à cette période sont rares bien que le pâturage pour des troupeaux ovins, soit ou ait été pratiqué dans de nombreuses régions européennes (GIBON et al., 1983), (BAKER \& CHARD, 1961; NEWTON \& JACKSON, 1985). Le plus souvent, seuls les arrières effets du pâturage sur la repousse de printemps sont étudiés (FrAME, 1970 ; WILMAN \& GRIFFITHS, 1978). En Nouvelle Zélande, PARMENTER \& BOSWELL (1983) ont analysé l'effet du nombre de pâtures pendant l'hiver sur la croissance à cette période. Les vitesses de croissance observées pour des rythmes d'exploitation mensuels vont de 5 à $15 \mathrm{~kg}$ de MS par ha et par jour en plein hiver avec des gelées mais on ne connaît pas précisément les températures.

Pour les parcelles B et $\mathrm{M}$, les rapports entre les vitesses de croissance (avant et après mi-février) pour l'ensemble du couvert végétal sont supérieurs ou égaux à ceux observés pour les longueurs de feuilles de dactyle (DURU, 1987a). On aurait pu s'attendre à l'inverse dans la mesure où les contrôles considérés pour le dactyle ont eu lieu en absence de défoliation. Ces différences peuvent s'interpréter par le développement parfois important de diverses espèces fourragères (comme le pissenlit) principalement en fin d'hiver.

\section{TABLEAU 6}

Moyennes ( $m$ ), écarts types ( $t$ ) des quantités de minéraux mobilisés (en $\mathrm{kg}$ ) et teneurs moyennes (\% MS) pour 3 hivers (81/82, $82 / 83,83 / 84)$.

Means $(\mathrm{m})$ and standard deviations $(\mathrm{t})$ of total mineral content $(\mathrm{kg})$ and mean content (\% DM) for 3 winters $(81 / 82,82 / 83,83 / 84)$.

\begin{tabular}{|c|c|c|c|c|}
\hline Traitement & Minéraux & $\underset{\mathrm{m}}{\text { Quantités }}$ & $\underset{t}{\text { mobilisées }}$ & Teneur moyenne \\
\hline \multirow[t]{3}{*}{ B0 } & $\mathbf{N}$ & 69,0 & 12,7 & 3,0 \\
\hline & $\mathbf{P}$ & 8,7 & 1,4 & 0,40 \\
\hline & $\mathrm{K}$ & 49,5 & 5,6 & 2,5 \\
\hline \multirow[t]{3}{*}{ Mo } & $N$ & 40,0 & 15,1 & 2,9 \\
\hline & $\mathrm{P}$ & 3,7 & 1,3 & 0,27 \\
\hline & $K$ & 27,7 & 7,5 & 1,7 \\
\hline \multirow[t]{3}{*}{$\mathrm{CO}$} & $N$ & 28,6 & 11,1 & 2,9 \\
\hline & $\mathrm{P}$ & 2,6 & 1,0 & 0,27 \\
\hline & $\mathrm{K}$ & 12,0 & 4,6 & 1,2 \\
\hline
\end{tabular}

Les quantités d'éléments minéraux mobilisés pour la croissance ont été mesurées à chaque prélèvement. Les bilans globaux sur la période hivernale sont très différents selon les parcelles (tabl. 6). Pour l'acide phosphorique et la potasse, il s'agit de différences de teneurs et de croissance, alors que pour l'azote il ne s'agit en première approximation que de différences de croissance. Ces résultats confirment le caractère limitant de la nutrition phospho-potassique pour les parcelles $M$ et $C$. La quantité totale d'azote fournie par le sol dépend du niveau de nutrition phosphopotassique.

\section{B. Comparaison aux situations réellement pâturées}

\section{Croissance}

L'intensité de la défoliation que nous avons pratiquée est le plus souvent supérieure à celle exercée par les animaux. En plein hiver, nous avons prélevé sur les parcelles pâturées entre 40 et $100 \mathrm{~kg}$ de MS par ha (8 observations) afin d'obtenir le même niveau de défoliation après prélèvement que sur les placettes destinées au contrôle de croissance. A la fin de la période de pâturage (avril), ces valeurs vont de 80 à $400 \mathrm{~kg}$ (8 observations). Les valeurs les plus élevées correspondent certainement à un décalage entre la croissance de l'herbe et la charge animale, surtout pour les parcelles de bonne fertilité. Ces données permettent de dire que les prélèvements réalisés dans les mises en défens sur-estiment les quantités qui ont été pâturées notamment au printemps. Par contre, nos défoliations étant plus sévères que celles des troupeaux, les vitesses de croissance immédiatement postérieures sont sans doute plus faibles que celles qui seraient observées sous pâturage continu (KING et al., 1984). Seules des études prenant en compte à la fois le troupeau et le couvert végétal permettraient de préciser ces hypothèses (HODGSON, 1985).

\section{Composition chimique}

La figure 6 compare les teneurs en azote total et en cellulose brute de l'herbe coupée (pour contrôle de croissance) et celles de l'herbe pâturée. On note une sigmoïde par rapport à la bissectrice. En début et milieu d'hiver, l'herbe pâturée est moins riche en azote que l'herbe coupée, et inversement en fin d'hiver. On peut noter que ces écarts sont différents selon les parcelles. A titre d'exemple, en fin d'hiver, les teneurs en azote de la parcelle B sont toujours supérieures en situation pâturée alors que pour les parcelles $M$ et $C$ elles sont voisines dans les deux situations. Ce constat nous conduit à formuler les hypothèses suivantes: en fin d'hiver, les animaux pâturent en moyenne des feuilles plus jeunes que celles observées dans les mises en défens puisque le pâturage est quotidien; l'importance relative des feuilles nouvellement apparues est d'ailleurs supérieure lorsque les conditions de nutrition minérale sont bonnes. En début et milieu d'hiver, le rythme d'apparition des feuilles est lent et la croissance est essentiellement constituée de feuilles d'âge élevé, pour lesquelles la sénescence est accélérée relativement à nos observations compte tenu du piétinement (TALLOWIN, 1985). La sélection alimentaire des animaux peut expliquer que ce qui reste sur pied soit de moins bonne valeur fourragère. Pour la cellulose, on peut adopter un raisonnement similaire mais le nombre plus faible de données ne nous permet pas d'être affirmatifs.

A partir des résultats d'analyse chimique, il est difficile d'évaluer la valeur alimentaire du pâturage dans la mesure où ces résultats sont le plus souvent en dehors des seuils pris en compte pour l'établissement des équations de prévision (ANDRIEU et WEISS, 1981 ; DEMARQUILly et al., 1981). Toutefois, on peut donner des ordres de grandeur en utilisant ces équations : nous obtenons des valeurs énergétiques et azotées variant de 0,80 à 1,20 UFL par $\mathrm{kg}$ de $\mathrm{MS}$ et de 80 à 

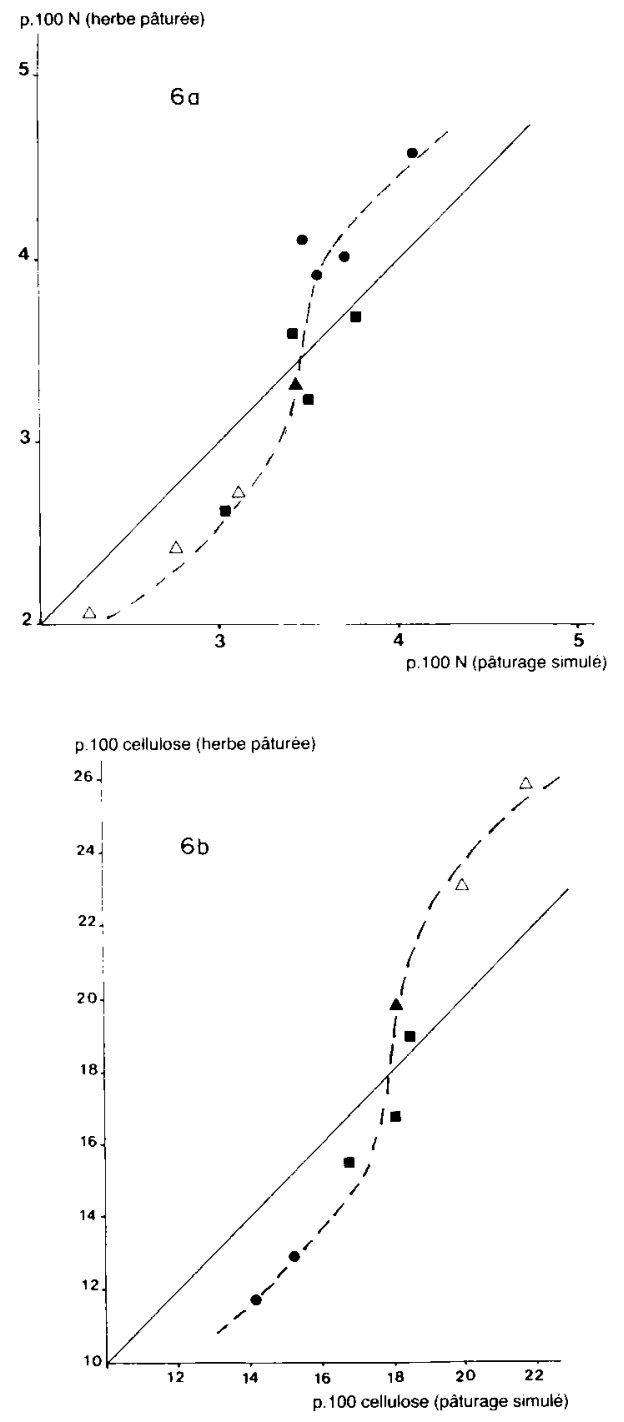

Figure 6

Comparaison de la composition chimique de l'herbe (p. $100 \mathrm{MS}$ ) des parcelles réellement pâturées à celles où le pâturage est simulé. $6 a$ azote total, $6 b$ cellulose brute.

Comparison of herbage chemical composition (\% DM) for plots really grazed and plots where grazing was simulated. $6 a$ total nitrogen, $6 b$ cellulose.

$230 \mathrm{~g}$ de MAD par $\mathrm{kg}$ de MS sur la base de la gamme de variations de la composition chimique indiquée figure 6 .

Ces résultats, à considérer avec précaution, montrent donc une très bonne valeur alimentaire et une forte variabilité (surtout pour l'azote) qu'il est possible de prévoir dans une certaine mesure.

\section{CONCLUSION}

\section{Maîtrise et prévision de la croissance hivernale et de sa qualité}

Les variations de vitesses de croissance observées en fin d'hiver selon les conditions de nutrition phosphopotassique et azotée, indiquent que des marges de progrès importantes sont possibles dans le milieu étudié, sachant que les parcelles de type $M$ et $C$ représentent au moins les trois quart des surfaces fauchées. Nous avons calculé précédemment (DuRU , 1985a) la date à laquelle la vitesse de croissance devenait supérieure ou égale à $30 \mathrm{~kg}$ par ha par jour. Nous observons des différences de 47 jours entre les parcelles $C$ et $B$, et de 59 jours avec une fertilisation azotée sur la parcelle $B$. Ces résultats montrent donc que la nutrition minérale aura un effet direct sur l'importance de la période d'affourragement du fait de la place plus ou moins importante du pâturage dans le rationnement. Par ailleurs, compte tenu des fortes variations entre années de la rigueur de l'hivernage (DURU \& CHARPENTEAU, 1981) l'apport d'azote en fin d'hiver pourrait permettre de pallier à des fins d'hivernage difficiles en augmentant les disponibilités au pâturage pour les mois de mars et avril. Connaissant la température et le niveau de fertilité d'une parcelle, il est désormais possible d'avoir une bonne estimation de la croissance. De même, on peut prévoir avec une précision satisfaisante les variations de la valeur alimentaire de l'herbe sur pied, dans les conditions d'un pâturage continu en cours de l'hiver.

D'un point de vue méthodologique, les interactions entre l'état du couvert végétal lors de la défoliation et la nutrition minérale demanderaient à être précisées. En effet, pour des parcelles portant des couverts végétaux différant par leur nutrition minérale, des défoliations à dates données correspondent à des indices foliaires et des hauteurs d'herbe différents. Il en résulte que les conditions de repousse ultérieure, notamment les niveaux de compétition pour la lumière et les minéraux, sont susceptibles d'être modifiées. Ainsi, on peut penser que les écarts observés entre situations de nutrition minérale différentes seraient moindres pour une croissance non perturbée par une défoliation. De manière plus générale, ce sont les interactions entre intensité de défoliation et nutrition minérale qu'il conviendrait d'approfondir puisque l'animal (HODGSON, 1985), de même que la nutrition minérale (DURU, 1987a), vont modifier, sans doute différemment, les composantes morphogénétiques de la croissance des graminées.

Reçu le 14 mai 1985. Accepté le $1^{\text {er }}$ octobre 1986.

\section{RÉFÉRENCES BIBLIOGRAPHIQUES}

Andrieu J., Weiss Ph., 1981. Valeur énergétique des fourrages verts in "Prévision de la valeur nutritive des aliments des ruminants". Ed. I.N.R.A. publications, route de St Cyr, 78000 Versailles, 61-80.

Baker H. K., Chard J. R. A., 1961. The production and utilization of winter grass at various centres in England and Wales, 1954-1960. J. Br. Grassl. Soc., 16, 185-189.
Demarquilly C., Grenet E., Andrieu J., 1981. Valeur azotée des fourrages in "Prévision de la valeur nutritive des aliments des ruminants". Ed. I.N.R.A. publications, route de St Cyr, 78000 Versailles, 129-154.

Duru M., 1983. Caractérisation de la pousse hivernale des prairies permanentes dans les Pyrénées Centrales. Agronomie, 5, 461-472. 
Duru M., 1985a. Winter and spring growth variability of grazed permanent meadow. The case of the Pyrenees Mountains. Proceedings of the $X V$ International Grassland Congress. Kyoto 24-31 aout, 595-597.

Duru M., 1985 $b$. Herbage dry matter yield and nitrogen content of a reproductive growth according to spring management. Case of permanent meadow in the Pyrenees Mountains. Proceedings of the $X V$ International Grassland Congress, Kyoto 24-31 août, 522-524.

Duru M., 1987. Croissance hivernale et printanière de prairies permanentes pâturées en montagne. I. Ecophysiologie du dactyle. Agronomie, 7 (1),

Duru M., Charpenteau J. L., 1981. The farming system in the Pyrenees. Elaboration of a model of constitution and utilization of hay stock. Ag. Syst, 7, 137-156.

Frame J., 1970. The effect of winter grazing by sheep on spring and early summer pasture production. J. Br. Grassl. Soc., 25, 167-171.

Gibon A., Duru M., Balent G., 1983. Ajustements entre les besoins $\mathrm{du}$ troupeau ovin et les rythmes de croissance de l'herbe. $8^{e}$ Journées de la Recherche Ovine et Caprine, I.N.R.A./ITOVIC, décembre Paris, 343-380.

Gibon A., Balent G., 1984. Mesure de l'ingestion des ovins et des bovins au pâturage hors domaine expérimental dans les Pyrénées Centrales. 5th Meeting of the European grazing group. Edinburgh, 2-5 octobre, $19 \mathrm{p}$.

Gillet M., 1980. Les graminées fourragères. Coll. « Nature et agriculture ». Gauthier-Villars, 306 pages.

Hodgson J., 1985. The significance of sward characteristics in the management of temperate sown pasture. Proceedings of the XV International Grassland Congress. Kyoto 24-31 août, 63-67.
King J., Grant S. A., Torvell L., Sim E. M., 1984. Growth rate, senescence and photosynthesis of ryegrass swards cut to maintain a range of values for leaf area index. Grass and Forage Science, 39, 371-380.

Newton J. E., Jackson C., 1985. The effects of management and duration of grazing by sheep during the autumn and winter on subsequent grass production. J. Agric., Camb., 105, 199-203.

Parmenter G. A., Boswell C. C., 1983. Effect of number and timing of winter grazings on winter and spring pasture production. New Z. J. of Exp. Agr., 11, 281-287.

Robson M. J., Deacon M. J., 1978. Nitrogen deficiency in small closed communities of S24 ryegrass. II. Changes in the weight and chemical composition of single leaves during their growth and death. Ann. Bot. 42, 1199-1213.

Salette J., Lemaire G., 1981. Sur la variation de la teneur en azote des graminées fourragères pendant leur croissance: formulation d'une loi de dilution. C.R. Acad. Paris, 292, série III, 875-878.

Salette J., Lemaire G., Robichet J., Huguet L., 1984. Teneurs en azote soluble du raygrass d'Italie semé en automne : évolution au cours de la croissance de printemps. Fourrages, 97, 3-16.

Tallowin J. R. B., 1985. Herbage losses from tiller pulling in a continuously grazed perennial ryegrass sward. Grass and Forage Science, 40, 13-18.

Wilman D., Griffiths P. D., 1970. The effect of winter and early spring grazing by sheep on subsequent sward production. J. agric. Sci. Camb., 90, 471-477. 